\title{
COEFICIENTES DE ANGSTRÖM-PRESCOTT E A EVAPOTRANSPIRAÇÃO DE REFERÊNCIA NA TRANSIÇÃO CERRADO-AMAZÔNIA DO MATO GROSSO
}

\author{
MARTIM, Charles Campoe - charlescampoemartim@gmail.com \\ Universidade Federal de Mato Grosso/ UFMT \\ ZAMADEI, Tamara - tamarazamadei@hotmail.com \\ Universidade Federal de Mato Grosso / UFMT
}

\author{
SOUZA, Adilson Pacheco de - pachecoufmt@gmail.com \\ Universidade Federal de Mato Grosso/ UFMT
ALMEIDA, Frederico Terra de - fredterr@gmail.com
Universidade Federal de Mato Grosso / UFMT

ZOLIN, Cornélio Alberto - cornelio.zolin@embrapa.br

Empresa Brasileira de Pesquisa Agropecuária - Agrossilvipastoril / EMBRAPA AGROSSILVIPASTORIL

\begin{abstract}
RESUMO: A radiação global $(H)$ e a duração do brilho solar ( $n$ ) são elementos meteorológicos que contribuem expressivamente para estudos agronômicos, ecológicos e hidrológicos. $\mathrm{Na}$ obtenção de $\mathrm{H}$ podem ser aplicados diferentes modelos de estimativas baseados em variáveis meteorológicas, todavia, a maneira mais simplificada baseia-se na correlação linear entre a transmissividade atmosférica e a razão de insolação. Objetivouse obter os coeficientes lineares (a) e angulares (b) da equação de Angström-Prescott e avaliar a sua aplicação nas estimativas de $\mathrm{H}$ e da evapotranspiração de referência (ETo), na região de Sinop, MT (transição Cerrado-Amazônia; $-11,86^{\circ} ;-55,48^{\circ}$ e $371 \mathrm{~m}$ de altitude), entre 2011 e 2016. Os dados diários foram agrupados em bases mensais, sazonais (estação do ano) e anuais. O coeficiente "a" para estimativas mensais de $\mathrm{H}$ variou de 0,21 a 0,32, enquanto que o coeficiente "b" variou de 0,41 a 0,49. A transmissividade atmosférica de $\mathrm{H}$ variou de $22,24 \%$ (novembro) a $76 \%$ (junho - estação seca). Os valores médios de $\mathrm{H}$ e ETo medidos e estimados nos agrupamentos foram de, 15,72 e $16,46 \mathrm{MJ} \mathrm{m}^{-2} \mathrm{dia}^{-1}$ e 2,90 e $3,05 \mathrm{~mm} \mathrm{dia}^{-1}$, respectivamente. Recomenda-se a utilização de $a=0,2441$ e $b=0,3774$ para estimativas de $\mathrm{H}$ pelos coeficientes da equação de Angstöm-Prescott, independentemente da época do ano.
\end{abstract}

PALAVRAS-CHAVE: transmissividade atmosférica, razão de insolação, radiação global, indicativos estatísticos.

\section{ANGSTRÖM-PRESCOTT COEFFICIENTS AND REFERENCE EVAPOTRANSPIRATION IN THE CERRADO-AMAZON TRANSITION REGION OF MATO GROSSO}

\begin{abstract}
The global radiation $(\mathrm{H})$ and the sunshine duration $(\mathrm{n})$ are meteorological elements that contribute significantly to agronomic, ecological and hydrological research's. In order to obtain $\mathrm{H}$, different models of estimates base on meteorological variables can be applied, however, the most simplified model way is based on the linear correlation between the atmospheric transmissivity and the insolation ratio. The objective of this study was to obtain the linear (a) and angular (b) coefficients of the AngströmPrescott equation and to evaluate its application in the estimates of $\mathrm{H}$ and reference evapotranspiration (ETo), in Sinop, Mato Grosso State, Cerrado-Amazon transition $\left(11.86^{\circ} \mathrm{S}, 55.48^{\circ} \mathrm{W}\right.$ and $371,0 \mathrm{~m}$ of altitude). The daily data were grouped into monthly, seasonal (season) and annual bases. The coefficient "a" for monthly $\mathrm{H}$ estimates ranged from 0.21 to 0.32 , while the "b" coefficient ranged from 0.41 to 0.49 . The atmospheric transmissivity of $\mathrm{H}$ ranged from $22.24 \%$ (November) to $76 \%$ (June - dry season). The mean values of $\mathrm{H}$ and ETo measured and estimated in the groups were 15.72 and 16.46
\end{abstract}


MJ $\mathrm{m}^{-2}$ day $^{-1}$ and 2.90 and $3.05 \mathrm{~mm} \mathrm{day}^{-1}$, respectively. It is recommended to use $\mathrm{a}=$ 0.2441 and $b=0.3774$ for $\mathrm{H}$ estimates by the coefficients of the Angström-Prescott equation, regardless of the time of year.

KEYWORDS: atmospheric transmissivity, sunshine duration, global radiation, statistical indicators.

\section{COEFICIENTES DE ANGSTRÖM-PRESCOTT Y LA EVAPOTRANSPIRACIÓN DE REFERENCIA EN LA TRANSICIÓN CERRADO-AMAZONIA DE MATO GROSSO}

RESUMEN: La radiación global $(\mathrm{H})$ y la duración del brillo solar $(\mathrm{n})$ son elementos meteorológicos que contribuyen expresamente a estudios agronómicos, ecológicos e hidrológicos. En la obtención de $\mathrm{H}$ se pueden aplicar diferentes modelos de estimaciones basadas en variables meteorológicas, sin embargo, la manera más simplificada se basa en la correlación lineal entre la transmisividad atmosférica y la razón de insolación. Se pretendió obtener los coeficientes lineales (a) y angulares (b) de la ecuación de Angström-Prescott y evaluar su aplicación en las estimaciones de $\mathrm{H}$ y de la evapotranspiración de referencia (ETo), en la región de Sinop, MT (transición CerradoAmazonia, $-11,86^{\circ},-55,48^{\circ}$ y $371 \mathrm{~m}$ de altitud), entre 2011 y 2016 . Los datos diarios se agruparon en bases mensuales, estacionales (estación del año) y anuales. El coeficiente "a" para las estimaciones mensuales de $\mathrm{H}$ varió de 0,21 a 0,32 , mientras que el coeficiente "b" varió de 0,41 a 0,49. La transmisividad atmosférica de $\mathrm{H}$ varió del 22,24\% (noviembre) al 76\% (junio - estación seca). Los valores medios de $\mathrm{H}$ y ETo medidos y estimados en las agrupaciones fueron de 15,72 y $16,46 \mathrm{MJ} \mathrm{m}^{-2}$ día $^{-1}$ y 2,90 y $3,05 \mathrm{~mm}$ día $^{-1}$, respectivamente. Se recomienda la utilización de $a=0,2441$ y $b=0,3774$ para estimaciones de $\mathrm{H}$ por los coeficientes de la ecuación de Angstöm-Prescott, independientemente de la época del año.

PALABRAS CLAVE: transmisividad atmosférica, razón de insolación, radiación global, indicativos estadísticos.

\section{COEFFICIENTS D'ANGSTRÖM-PRESCOTT ET ÉVAPOTRANSPIRATION DE RÉFÉRENCE DANS LA TRANSITION CERRADO-AMAZONIENNE DU MATO GROSSO}

RESUME: Le rayonnement global $(\mathrm{H})$ et la durée de la luminosité solaire $(\mathrm{n})$ sont des éléments météorologiques qui contribuent de manière significative aux études agronomiques, écologiques et hydrologiques. Pour obtenir le $\mathrm{H}$, différents modèles d'estimations basés sur des variables météorologiques peuvent être appliqués. Cependant, la manière la plus simplifiée est basée sur la corrélation linéaire entre la transmissivité atmosphérique et le rapport d'insolation. L'objectif était d'obtenir les coefficients linéaires (a) et angulaires (b) de l'équation d'Angström-Prescott et d'évaluer son application à l'estimation $\mathrm{du} \mathrm{H}$ et à l'évapotranspiration de référence (ETo) dans Sinop, MT. $-11,86^{\circ},-55,48{ }^{\circ}$ et $371 \mathrm{~m}$ d'altitude) entre 2011 et 2016 . Les données quotidiennes ont été regroupées en bases mensuelles, saisonnières (saison) et annuelles. Le coefficient "a" pour les estimations mensuelles de mercure variait de 0,21 à 0,32, tandis que le coefficient "b" allait de 0,41 à 0,49. La transmissivité atmosphérique du mercure variait de $22,24 \%$ (novembre) à $76 \%$ (juin - saison sèche). Les valeurs moyennes de $\mathrm{H}$ et de ETo mesurées et estimées dans les groupes étaient respectivement de 15,72 et $16,46 \mathrm{MJ} \mathrm{m}^{-2}$ jour $^{-1}$ et de 2,90 et $3,05 \mathrm{~mm}$ jour ${ }^{-1}$. Il est recommandé d'utiliser $a=0.2441$ et $b=0.3774$ pour les estimations de $\mathrm{H}$ par les coefficients de l'équation de Angstöm-Prescott, quelle que soit la période de l'année.

MOTS-CLES: transmissivité atmosphérique, taux d'insolation, rayonnement global, statistique indicative.

\section{INTRODUÇÃO}

A extensão territorial e distribuição dos biomas brasileiros, aliado aos avanços das tecnologias nos setores energético, agropecuário e ambiental, tem criado demandas crescentes pelo conhecimento da disponibilidade de radiação 
solar (BIUDES et al., 2015; ANDRADE e TIBA, 2016; ROSSI et al. 2018). Essa variável meteorológica permite aplicações em projetos de aproveitamento de energia, meteorologia/climatologia, química da atmosfera, balanços de energia e hídrico, qualidade do ar e interação clima/oceano, consumo hídrico de plantas, modelos de desenvolvimento e produção de culturas, conforto térmico na arquitetura e na construção civil, dentre outros (SOUZA et al., 2016; SILVA et al. 2017).

A radiação solar após atingir o topo da atmosfera terrestre, de maneira simplificada, pode sofrer atenuação atmosférica pelos fenômenos da reflexão, absorção e difusão em virtude da interação das ondas eletromagnéticas com os gases constituintes da atmosfera, nuvens e/ou com partículas em suspensão (PALÁCIOS et al., 2018; ROSSI et al., 2018). Denomina-se radiação global, a quantidade de fluxos energéticos que atingem a superfície terrestre e que podem ser resultantes das componentes direta, difusa e refletida, dependendo da inclinação da superfície (SOUZA e ESCOBEDO, 2013).

A maioria das estações meteorológicas brasileiras monitora de rotina apenas a irradiação global $(H)$ em função dos custos de aquisição e operação dos sensores para medidas das radiações espectrais e da direta na incidência. Todavia, apesar da evolução de redes meteorológicas no Brasil, quando comparado com outras variáveis meteorológicas, as medidas de $\mathrm{H}$ são mais escassas, e mesmo quando disponíveis, são limitadas quanto ao período (anos), pois dependeram do avanço da instrumentação meteorológica. Nesse sentido, modelos de estimativa ainda são importantes por permitirem a obtenção de séries temporais longas, preenchimento de falhas e analises de sazonalidades (LIU et al., 2014; BAYRAKÇL et al., 2017).

Na estimativa da radiação solar, normalmente são empregados dois tipos de modelos: estatísticos (ou de decomposição) e parametrizados (SOUZA et al., 2011). Os modelos estatísticos são representados por equações ajustadas por meio de correlações com a razão de insolação e/ou com as frações radiométricas. Já os modelos parametrizados carecem de outras informações das condições atmosféricas locais, conforme a componente a ser estimada, ou então a utilização de percentuais fixos de ocorrência de cada componente da radiação global.

Angström (1924) propôs um modelo de estimativa de $\mathrm{H}$ baseado na correlação linear entre a transmissividade atmosférica (H/HO - radiação global e radiação no topo da atmosfera) e a razão de insolação ( $\mathrm{n} / \mathrm{N}$ insolação/fotoperíodo) para dias sem nuvens. Posteriormente, em função das dificuldades da obtenção da radiação sem a interferência das nuvens (dias de céu aberto), foram propostas alterações no modelo inicial (Prescott 1940), no qual a influência das nuvens em $(\mathrm{H} / \mathrm{HO})$ foi expressa por um modelo linear conhecido como Agström-Prescott $(\mathrm{H} / \mathrm{Ho}=\mathrm{a}+\mathrm{b}(\mathrm{n} / \mathrm{N}))$, no qual os coeficientes "a" e "b" devem ser ajustados para cada região (SOUZA e ESCOBEDO, 2013; PAULESCU et al., 2016; SILVA et al., 2017).

Dentre as possibilidades físicas desse modelo, tem-se duas situações: i) quando $\mathrm{n} / \mathrm{N}$ for nulo (céu nublado), o coeficiente " $\mathrm{a}$ " indica a transmissividade atmosférica mínima de $\mathrm{H}$; ii) quando $\mathrm{n} / \mathrm{N}$ tende ao valor unitário, o coeficiente "b" mostra a relação de crescimento de $\mathrm{H} / \mathrm{Ho}$, em conjunto, indicam a transmissividade máxima de H (DALLACORT et al., 2004; DORNELAS et al., 2006; PAULESCU et al., 2016; SILVA et al., 2017). Vários fatores locais 
influenciam nesses coeficientes, como nebulosidade, densidade óptica atmosférica, época do ano, longitude, latitude, altitude, dentre outros (DORNELAS et al., 2006; LIU et al., 2009; SOUZA et al., 2016c). Em geral, o efeito do coeficiente "a" tende a ser mais significativo quando comparado com o coeficiente " $b$ " na estimativa de H (LIU et al., 2014; PAULESCU et al., 2016).

Tanto a energia radiante incidente em ondas curtas $(H)$, quanto a duração do brilho solar $(n)$, influenciam diretamente nas interações físicas, químicas e biológicas das plantas, com destaques para a fotossíntese e a evapotranspiração (CARVALHO et al., 2011; SOUZA et al., 2016a; SOUZA et al., 2017). Ao longo do tempo, a busca por padrões/referências nas avaliações de perdas de água por vegetações, permitiram a inserção do conceito de evapotranspiração de referência (ETo), que indica a perda de água de uma vegetação que cobre completamente o solo, sem restrições hídricas, nutricionais ou problemas fitossanitários; quando essas condições ocorrem a vegetação expressa o seu potencial produtivo/genético para as condições ambientais locais.

Muitos modelos de estimativas da ETo foram desenvolvidos para diferentes regiões e condições climáticas, todavia, a proposição final do modelo Penman-Monteith FAO 56 foi a que mais se aproximou de valores obtidos por lisímetros de pesagem e passou a ser amplamente recomendada (ALLEN et al., 1998; CARVALHO et al., 2016). Como cada região brasileira, apresenta características atmosféricas diferenciadas ao longo do ano, vários trabalhos foram desenvolvidos para estimativa de $\mathrm{H}$ por modelos simplificados, com destaques para os trabalhos BLANCO e SENTELHAS (2002) para Piracicaba, SP; DALLACORT et al. (2004) em Pelotina, PR; DORNELAS et al. (2006) em Brasília, DF; CARVALHO et al. (2011) em Seropédica, RJ; SOUZA e ESCOBEDO (2013) e SILVA et al. (2017) para Botucatu-SP; SOUZA et al. (2017) em vários municípios do Mato Grosso, dentre outros. Todavia, estudos com as aplicações dessas estimativas na obtenção da ETo são mais escassas (DORNELAS et al., 2006; LIU et al., 2009; CARVALHO et al., 2011; TANAKA et al., 2017).

Para Mato Grosso, em especial na região do município de Sinop (-ecótono Cerrado-Amazônia), Souza et al. (2017) avaliaram modelos de estimativa de H com base na temperatura e umidade relativa do ar, todavia, os erros gerados pela aplicação dessas estimativas de $\mathrm{H}$ na ETo ainda foram elevados (SOUZA et al., 2016a). Tanaka et al. (2016) avaliaram diferentes modelos de estimativa de ETo, todavia, nenhum modelo baseou-se na insolação como forma de obtenção de $H$ e do saldo de radiação, e tão pouco distribuído em diferentes agrupamentos de dados (mensal e sazonal). Nesse sentido, ainda existem lacunas para estudos das interações entre H e ETo no Mato Grosso.

Aliado a isso, o Centro-Norte mato-grossense é responsável por grande parte dos grãos produzidos no estado, e consequentemente o conhecimento da evapotranspiração assume fundamental importância para o planejamento de sistemas hidroagrícolas e para os plantios de sequeiro. Por conseguinte, objetivou-se obter os coeficientes da equação de Angström-Prescott e avaliar as suas aplicações nas estimativas da radiação global e da evapotranspiração de referência, em Sinop, ecótono Cerrado-Amazônia no estado de Mato Grosso. 


\section{MATERIAL E MÉTODOS}

Os valores dos elementos meteorológicos foram obtidos da base de dados da Estação Meteorológica Automática (EMA) do Campus Universitário de Sinop, da Universidade Federal de Mato Grosso - UFMT (latitude $-11,86^{\circ}$, longitude $-55,48^{\circ}$, altitude $371 \mathrm{~m}$ ), no período de 2011 a 2016 . O clima da região segundo a classificação climática de Köppen, é do tipo Aw (tropical quente e úmido), caracterizado por duas estações bem definidas: chuvosa (outubro a abril) e seca (maio a setembro). As temperaturas médias mensais variam de 23,0 a $25,8{ }^{\circ} \mathrm{C}$, com precipitações anuais em torno de $1974 \mathrm{~mm}$ (Souza et al. 2013) e radiação global média de Sinop variando de 16,36 a 21,26 MJ m-2 d-1 de julho e agosto (SOUZA et al., 2016 b).

Na Figura 1 observa-se a área de influência da estação avaliação, sendo considerado um raio de $250 \mathrm{~km}$, com fitofisionomia e relevo similares.

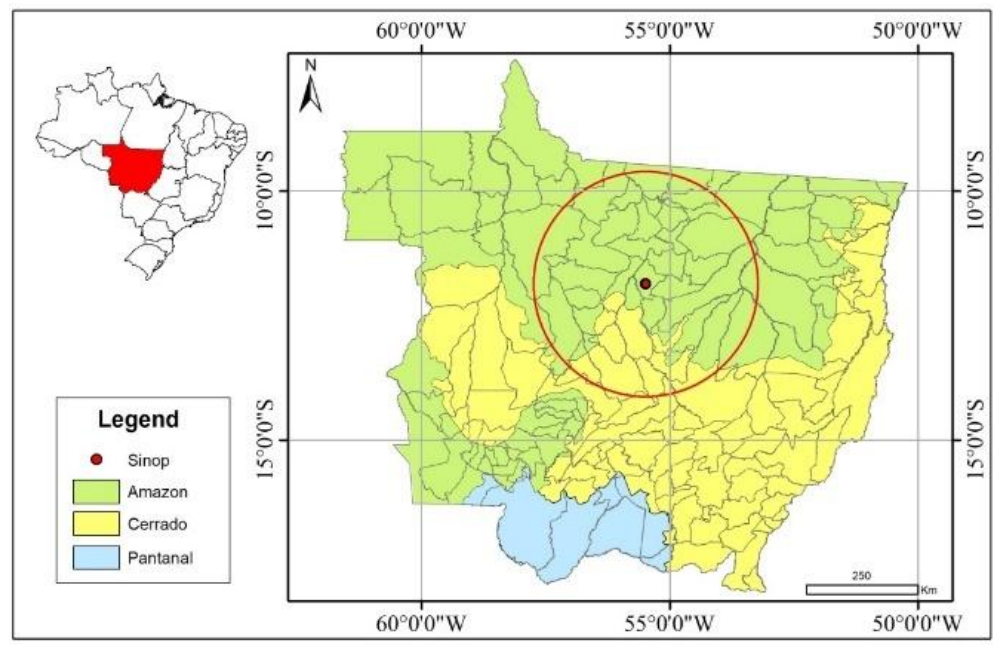

Figura 1 - Localização da estação meteorológica em Sinop-MT e dos biomas matogrossenses.

A irradiância global (G) foi monitorada por piranômetro CM3 da Kipp e Zonen, posicionados a $1 \mathrm{~m}$ de altura, com sensibilidade de resposta de $\pm 10-35$ $\mu \mathrm{V} / \mathrm{Wm}-2$, tempo de resposta de $18 \mathrm{~s}$, resposta a temperatura de $\pm 1,0 \%$ para a faixa de -40 a $80^{\circ} \mathrm{C}$ e desvios para o efeito cosseno de $\pm 2 \%\left(0<z<80^{\circ}\right.$. $\mathrm{Na}$ aquisição dos dados foi utilizado um datalogger CR1000 (Campbell Scientific) operando na frequência de $1 \mathrm{~Hz}$ e armazenando médias de $5 \mathrm{~min}$. Nesse caso, as unidades e símbolos seguem as recomendações do Comitê da International Solar Energy Society (ISES, 1997).

Para medição das demais variáveis meteorológicas, a EMA possui os seguintes sensores: velocidade e direção do vento (anemômetro, 03002-L RM Young) a $10 \mathrm{~m}$ de altura, psicrômetro com abrigo termométrico (Vaisala, CS 215) a $2 \mathrm{~m}$ de altura, pluviógrafo (Vaisala, TE 525) a 1,5 m de altura, aliado a isso, ainda ocorrem as medidas diárias de rotina da insolação com um heliógrafo Campbell-Stockes a 1,5 m de altura, evaporação com Tanque Classe A e altura pluviométrica com um pluviômetro Ville de Paris.

Foram aplicadas as considerações de Iqbal (1983) para obtenção do fotoperíodo $(\mathrm{N})$ e da radiação no topo da atmosfera $\left(\mathrm{H}_{0}\right)$. Realizou-se a filtragem da base de dados diária, considerando-se os critérios de $\mathrm{H} / \mathrm{H}_{0}<0,82$; e ii) $\mathrm{n}<$ 
N. Os valores dos coeficientes " $a$ " e " $b$ " da equação de Angström-Prescott (Eq. 1) foram calibrados através de correlações lineares entre a transmissividade atmosférica $\left(\mathrm{H} / \mathrm{H}_{0}\right)$ e a razão de insolação $(\mathrm{n} / \mathrm{N})$, nos agrupamentos mensal, sazonal e anual. Foram utilizados os dados de 2011 a 2014 e 2016) para calibração dos coeficientes (a, b), enquanto que os dados de 2015 foram usados na avaliação do desempenho estatístico (validação).

$$
H=\left(a+b \frac{n}{N}\right) H_{0}
$$

em que: $\mathrm{H}$ - irradiação global diária $\left(M J \mathrm{~m}^{-2} \mathrm{~d}^{-1}\right) ; \mathrm{H}_{0}$ - irradiação no topo da atmosfera diária ( $\left.\mathrm{MJ} \mathrm{m} \mathrm{m}^{-2} \mathrm{~d}^{-1}\right)$; $\mathrm{a}$ e $\mathrm{b}$ - constantes do modelo; $\mathrm{n}$ - número de horas de brilho solar $\left(\mathrm{h} \mathrm{d}^{-1}\right)$; $\mathrm{N}$ - número máximo de horas de brilho solar $\left(\mathrm{h} \mathrm{d}^{-}\right.$ $\left.{ }^{1}\right)$.

A evapotranspiração de referência (ETO) foi obtida pelo método de Penman Monteith Fao 56 (Eq. 2), que apresenta dependência do saldo de radiação (Rn). Pelas recomendações de Allen et al. (1998) e Pereira et al. (2013), a obtenção de Rn pode ser dada por estimativas das componentes dos balanços de radiação em ondas curtas (BOC) e em ondas longas (BOL). A obtenção de BOL de maneira simplificada comumente é realizada pelo modelo Brunt, enquanto BOC é obtido pela diferença entre $(H)$ e a radiação refletiva (dependente do albedo da vegetação).

Dessa forma, avaliou-se as aplicações de $\mathrm{H}$ estimada pelos coeficientes de Angstrom-Prescott calibrados, na obtenção de BOC e Rn, e consequentemente ETo, tendo nesse caso, $\mathrm{H}$ medida como referência.

$$
\mathrm{ET}_{0}=\frac{0,408 \Delta\left(\mathrm{R}_{\mathrm{n}}-\mathrm{G}\right)+\left(\gamma \frac{900}{\overline{\mathrm{T}}+275}\right) \mathrm{u}_{2}\left(\mathrm{e}_{\mathrm{s}}-\mathrm{e}\right)}{\Delta+\left[\gamma\left(1+0,34 \mathrm{u}_{2}\right)\right]}
$$

em que: $\quad \mathrm{ET}_{0}=$ evapotranspiração de referência $\left(\mathrm{mm} \mathrm{dia}^{-1}\right) ; \mathrm{Rn}=$ Saldo de

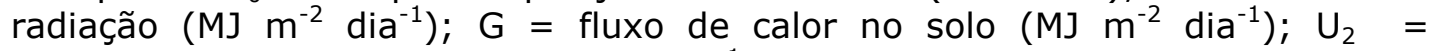
velocidade do vento a $2 \mathrm{~m}$ e altura $\left(\mathrm{m} \mathrm{s}^{-1}\right)$; es = pressão de saturação do vapor d'água do $\operatorname{ar}(\mathrm{kPa}) ; \mathrm{e}=$ pressão do vapor d 'água do $\operatorname{ar}(\mathrm{kPa}) ; \Delta=$ inclinação da curva da pressão de vapor saturado versus temperatura $\left(\mathrm{kPa}{ }^{\circ} \mathrm{C}^{-1}\right)$; UR = umidade relativa do ar (\%); $\lambda=$ calor latente de evaporação; $\mathrm{Y}=$ coeficiente psicrométrico $\left(\mathrm{kPa}^{\circ} \mathrm{C}^{-1}\right)$. (ALLEN et al., 1998; CARVALHO et al., 2011; SOUZA et al., 2016a)

Para avaliação do desempenho estatístico dos coeficientes calibrados, foram avaliados os valores estimados e medidos de $\mathrm{H}$ e de ETo, por meio do emprego dos seguintes indicativos estatísticos: MBE (Mean Bias Error), RMSE (Root Mean Square Error) e índice de ajustamento "d" de Willmott et al. (1985) descrito nas equações 3, 4 e 5, (Souza et al. 2016a, Tanaka et al. 2016).

$M B E=\frac{\sum_{i=1}^{N}(P i-O i)}{N}$ 


$$
\begin{aligned}
& \text { RMSE }=\left[\frac{\sum_{i=1}^{N}(\mathrm{Pi}-\mathrm{Oi})^{2}}{\mathrm{~N}}\right]^{\frac{1}{2}} \\
& \mathrm{~d}=1-\frac{\sum_{\mathrm{i}=1}^{\mathrm{N}}(\mathrm{Pi}-\mathrm{Oi})^{2}}{\sum_{\mathrm{i}=1}^{N}\left(\left|\mathrm{P}^{\prime} \mathrm{i}\right|+\left|\mathrm{O}^{\prime} \mathrm{i}\right|\right)^{2}}
\end{aligned}
$$

em que: $\mathrm{Pi}$ - valores estimados; Oi - valores medidos; $\mathrm{N}$ - número de observações; $\left|P^{\prime} i\right|^{-}$valor absoluto da diferença $P i-\overline{O i} ;\left|O^{\prime} i\right|-$ valor absoluto da diferença $\mathrm{Oi}-\overline{\mathrm{O} i}$.

\section{RESULTADOS E DISCUSSÃo}

Entre maio e setembro, independentemente do ano, ocorrem menores variações de $\mathrm{H}$ em função da maior estabilidade da transmissividade atmosférica (cobertura de céu com baixa nebulosidade - estação seca regional) (Figura 2). O maior e o menor valor de radiação encontrado na série histórica foi de 27,22 e

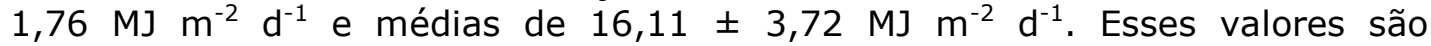
semelhantes às observações de Souza et al. (2016) para a região Amazônica de Mato Grosso, no qual, entre abril e setembro (estação seca da região), ocorrem maiores índices de transmissividade atmosférica e menores oscilações de $\mathrm{H}$, decorrentes da baixa atenuação da radiação solar pela interação com os constituintes atmosféricos. Já durante a estação chuvosa, em geral, os valores de Kt são menores, tendo em vista que nesta época a concentração de nuvens e vapor d'água na atmosfera é a maior do ano.

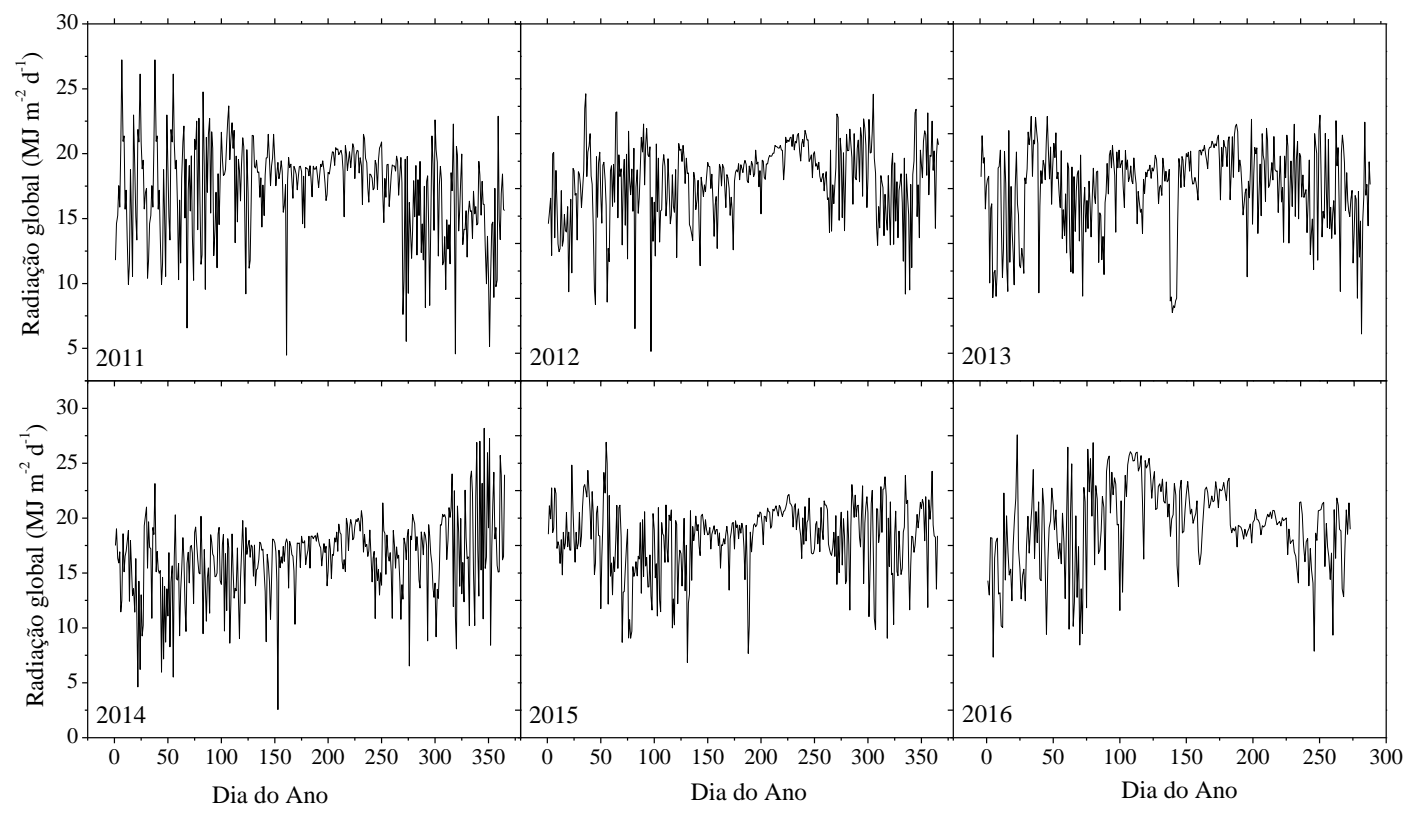

Figura 2 - Variação anual da radiação global diária (H), entre 2011 e 2016, em SinopMT. 
A correlação em agrupamento total de dados apresentou coeficiente de determinação $\left(R^{2}\right)$ de 0,7285 (Figura 3). Nesse caso, a transmissividade atmosférica variou de 24,42 a $62,16 \%$, pela análise dos coeficientes linear e angular. Para os demais agrupamentos de dados, os coeficientes calibrados são apresentados na Tabela 1. Destaca-se nesse caso, que em todos os meses, os coeficientes de determinação ajustados foram significativos a $1 \%$ de probabilidade.

Comparando-se com os valores propostos por Allen et al. (1998), nos quais os coeficientes "a" e "b" são iguais a $0,29 * \cos$ (latitude) e 0,52, respectivamente, foram obtidos valores inferiores de "b" em todos agrupamentos de dados avaliados, com alterações significativas nos valores de "a" ao longo dos meses. Esse comportamento indica a necessidade de calibrações locais dos coeficientes de Angström-Prescott, para evitar erros significativos nas aplicações que dependam de $\mathrm{H}$.

Os maiores valores de R2 foram obtidos na transição da estação secachuvosa (setembro, outubro e novembro), com 0,6587; 0,7732 e 0,7519; enquanto que, o menor valor de $r^{2}$ foi obtido em julho $(0,3133)$. Segundo Blanco e Sentelhas (2002), Paulescu et al. (2016) e Silva et al. (2017), valores de $\mathrm{R}^{2}$ para esse tipo de correlação no inverno é menor quando comparado a outras estações, devido as variações nas espessuras de nuvens e nas concentrações de aerossóis ao longo do período seco.

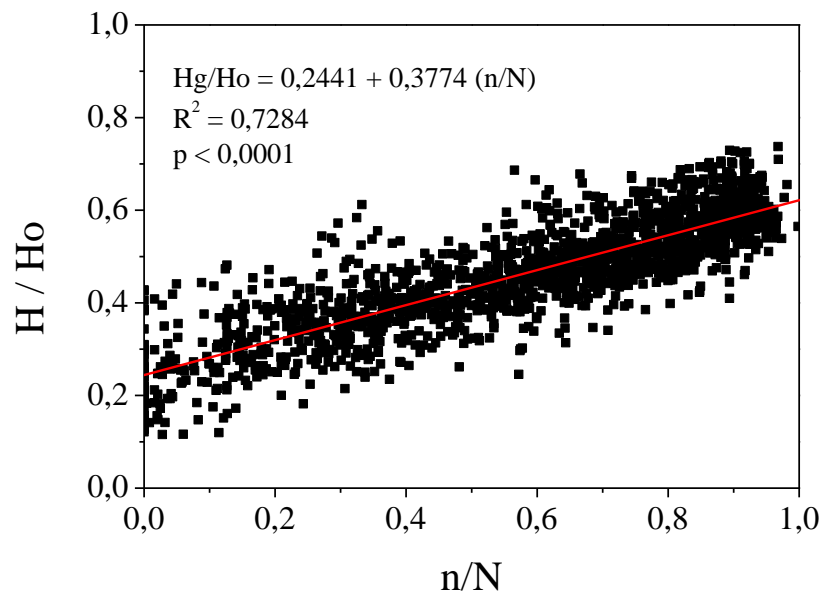

Figura 3 - Correlação entre a razão de insolação $(n / N)$ e transmissividade atmosférica (H/Ho), em agrupamento total de dados (5 anos), em Sinop-MT.

Tabela 1 - Coeficientes angulares (a) e lineares (b) da equação de Angstrom-Prescott, em agrupamentos de dados mensais e sazonais, em Sinop-MT.

\begin{tabular}{lccccc}
\hline \multicolumn{1}{c}{ Período } & $\mathrm{a}$ & $\mathrm{b}$ & $\mathrm{r}^{2}$ & Tmax & Tmin \\
\hline Janeiro & 0,2429 & 0,3352 & 0,5306 & 57,81 & 24,29 \\
Fevereiro & 0,2523 & 0,3098 & 0,5673 & 56,21 & 25,23 \\
Março & 0,2692 & 0,3491 & 0,5727 & 61,83 & 26,92 \\
Abril & 0,2254 & 0,4439 & 0,7070 & 66,93 & 22,54 \\
Maio & 0,2776 & 0,3339 & 0,5154 & 61,15 & 27,76 \\
Junho & 0,2363 & 0,4314 & 0,3899 & 66,77 & 23,63 \\
Julho & 0,3139 & 0,3096 & 0,3133 & 62,35 & 31,39
\end{tabular}




\begin{tabular}{llllll} 
Agosto & 0,2250 & 0,4005 & 0,4573 & 62,55 & 22,50 \\
Setembro & 0,2423 & 0,3337 & 0,6587 & 57,60 & 24,23 \\
Outubro & 0,2485 & 0,3277 & 0,7732 & 57,62 & 24,85 \\
Novembro & 0,2224 & 0,4039 & 0,7519 & 62,63 & 22,24 \\
Dezembro & 0,2779 & 0,3489 & 0,5441 & 62,68 & 27,79 \\
\hline Primavera & 0,2459 & 0,3432 & 0,7235 & 58,91 & 24,59 \\
Verão & 0,2318 & 0,3674 & 0,6906 & 59,92 & 23,18 \\
Outono & 0,2459 & 0,4003 & 0,6833 & 64,62 & 24,59 \\
Inverno & 0,2464 & 0,3753 & 0,5033 & 62,17 & 24,64 \\
\hline
\end{tabular}

Tmax e Tmin: transmissividade atmosférica máxima e mínima estimada, respectivamente.

Nesse caso, observa-se a importância da calibração regional dos coeficientes da equação de Angstrom-Prescott, visto que em geral, pelas recomendações de Allen et al. (1998), seriam usados para a região de Sinop-MT os valores de 0,2838 e 0,52 para os coeficientes "a" e "b", respectivamente, ou seja, seriam geradas superestimativas de $\mathrm{H}$ e em todas as suas possibilidades de aplicação (como a evapotranspiração). Liu et al. (2009), utilizando os coeficientes propostos pela FAO em comparação aos ajustados localmente, para estimar $H$, verificaram que diferentes formas de obtenção de $H$ permitiram superestimativas de 3 a $15 \%$ da ETo calculada por Penman-Monteith. Campelo Júnior (1998) encontrou para Santo Antônio do Leverger - MT, um coeficiente

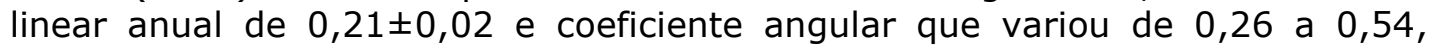
corroborando com este trabalho. Já Silva et al. (2017) para Botucatu-SP obtiveram em agrupamento anual de dados, valores de "a" e "b" de 0,253 e 0,465 , respectivamente.

No agrupamento mensal, a maior transmissividade de $\mathrm{H}$ foi estimada para junho $(76 \%)$ e a menor em novembro $(22,24 \%)$, períodos seco e chuvoso, respectivamente. Esse padrão também foi observado no agrupamento sazonal, com maior transmissividade no outono $(64,62 \%)$ e menor no verão $(23,18 \%)$. A transmissividade atmosférica de $\mathrm{H}$ é influenciada por aerossóis, vapor de água e concentrações de nuvens, que apresentam variabilidade dependente das condições climáticas em mesoescala (ÖZTOPAL e SEN, 2003; CHAÂBANE et al., 2004; DALLACORT et al., 2004; DORNELAS et al., 2006; SOUZA et al., 2016b-c; SILVA et al., 2017; PALÁCIOS et al., 2018).

Em estudo comparando o uso de diferentes modelos simplificados de estimativa de $\mathrm{H}$, Bayrakçl et al. (2017) verificaram que nos modelos polinomiais cúbico e quadrático com aplicações da razão de insolação $(n / N)$, foram obtidos melhores desempenho estatísticos em janeiro-junho e julho-dezembro respectivamente, sendo que neste caso, os modelos lineares (correspondentes ao avaliado nesse trabalho), provocaram menores desempenhos de ajuste em alguns meses. Todavia, Liu et al. (2014), aumentando a complexidade de modelos para a estimativa de $\mathrm{H}$, não encontraram melhorias significativas em correlações polinomiais com n/N. Souza et al. (2016c) relataram que, dentre os vários modelos avaliados, o polinomial de primeiro grau foi o recomendado devido a sua simplicidade e desempenho.

A fim de avaliar as causas de variação dos coeficientes ajustados, principalmente na estação seca da região, analisou-se a frequência de ocorrência da razão de insolação (Figura 4), evidenciando que de outubro a abril, $\mathrm{n} / \mathrm{N}$ apresentou variação entre 0 e 1,0. Já entre maio e setembro, as 
frequências de ocorrências de $\mathrm{n} / \mathrm{N}$ foram de 29,$6 ; 30,1 ; 46,6 ; 39,3$ e $25,9 \%$ para classes de $\mathrm{n} / \mathrm{N}$ de $0,8-0,9 ; 0,8-0,9 ; 0,9-1,0 ; 0,8-0,9$ e $0,7-0,8$, respectivamente.

Segundo Öztopal e Sen (2003) as chuvas causam uma dispersão nos valores dos coeficientes ajudados em regressões lineares, diminuindo assim o coeficiente de determinação. Pelas Figuras 4 e 5 , no início e final do ano (estação chuvosa), a frequência de ocorrência de $\mathrm{n} / \mathrm{N}$ apresenta-se mais distribuída em diferentes classes no histograma, enquanto nos meses da estação seca, a distribuição tem picos indicando a máxima razão de insolação e transmissividade atmosférica.

A frequência dos valores do coeficiente de transmissividade atmosférica (Kt) (Figura 5) indica comportamento inverso ao de $n / N$, no qual, independentemente do período, ocorre distribuição normal. Nos meses de junho, julho e agosto, a maior frequência observada foi de 33,$73 ; 49,42$ e $38,76 \%$ para Kt entre $0,5-0,6$, respectivamente. Na região amazônica do Mato Grosso, Souza et al. (2016b) encontraram maiores valores de Kt entre abril e setembro, sendo que a sazonalidade anual dessa fração radiométrica é dependente principalmente das concentrações de vapor d'água e aerossóis (Palácios et al. 2018).

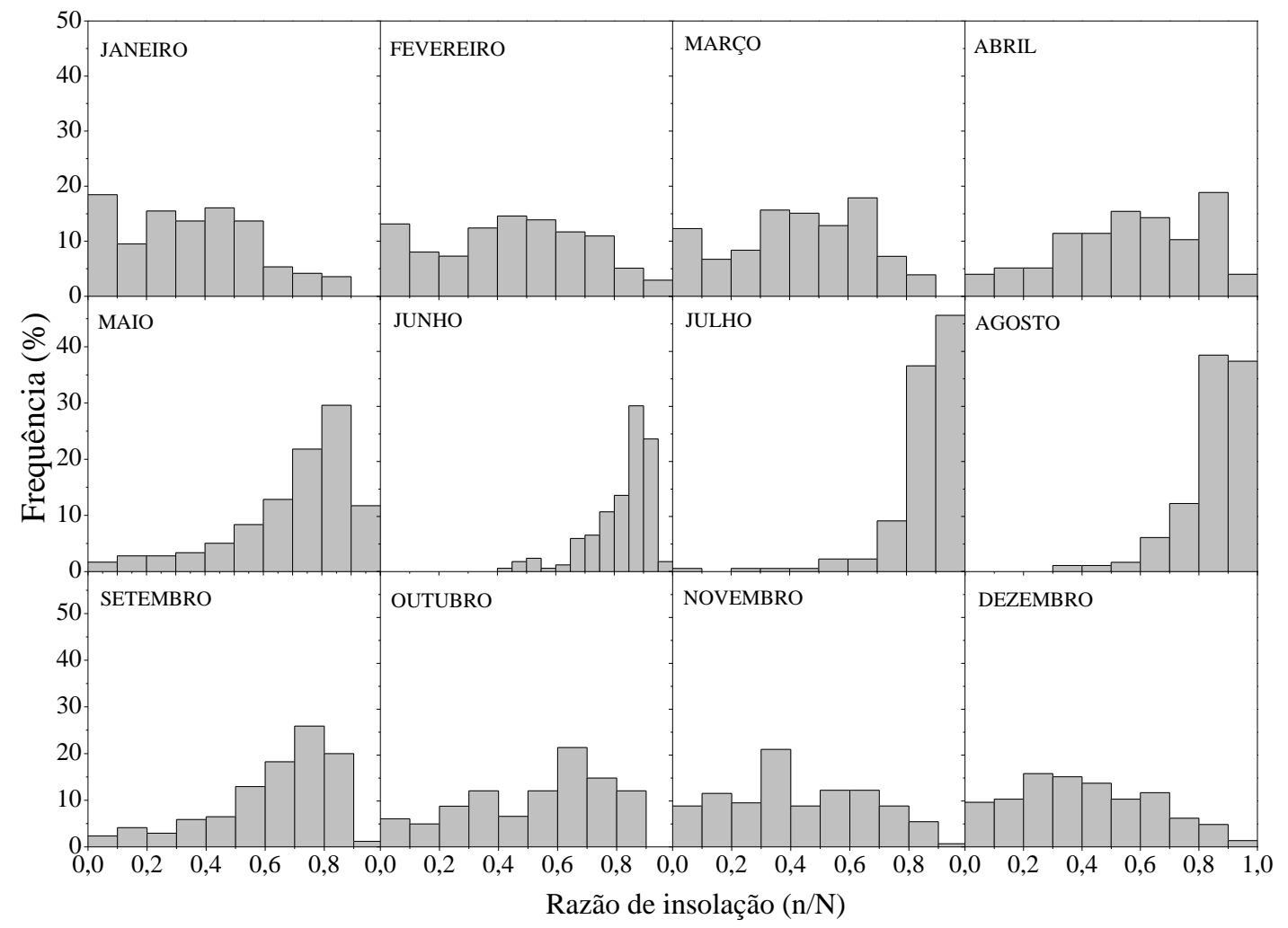

Figura 4 - Distribuição de frequência mensal da razão de insolação (n/N), entre 2011 a 2016, em Sinop-MT. 


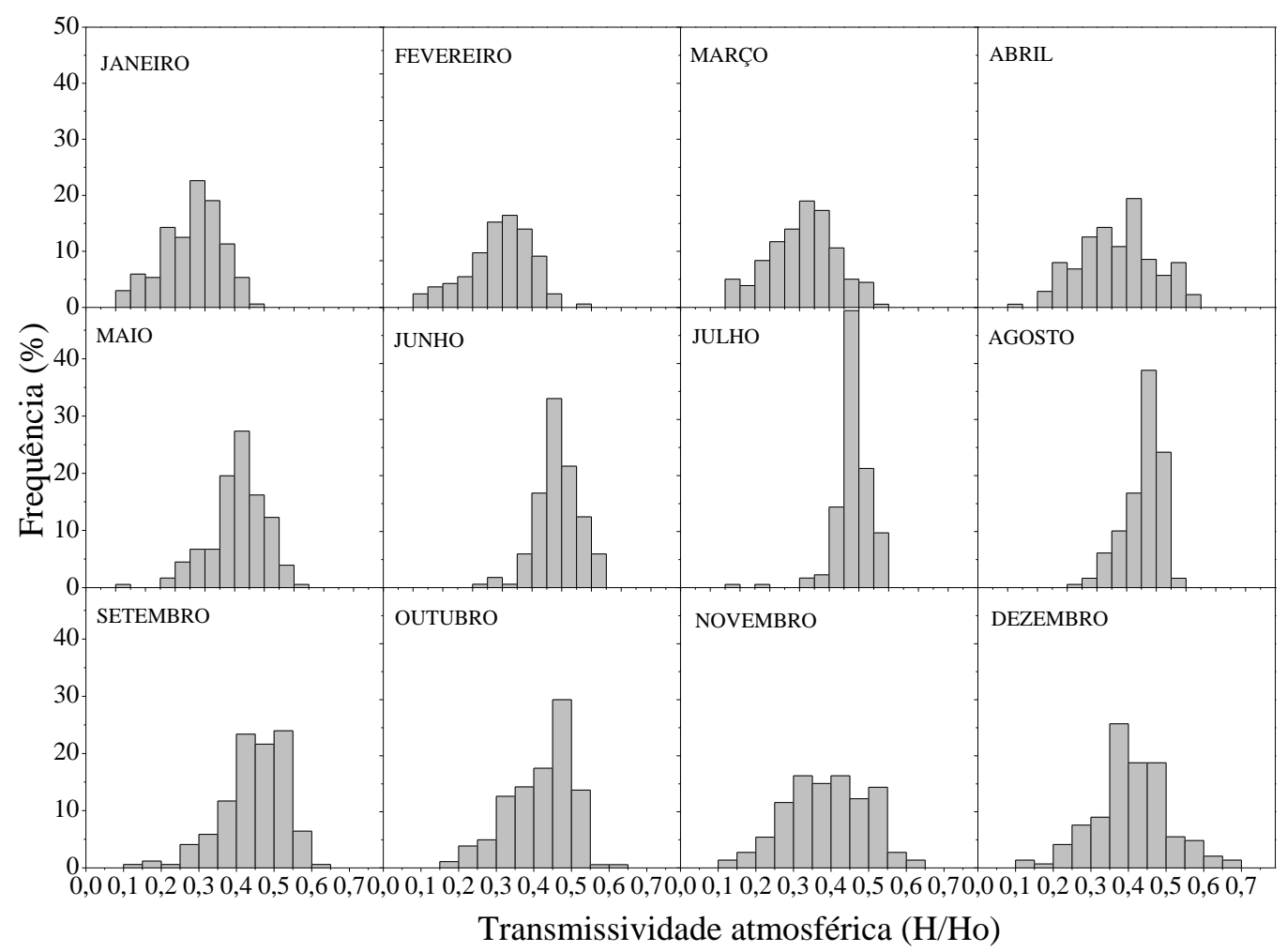

Figura 5 - Distribuição de frequência mensal do coeficiente de transmissividade atmosférica (Kt), entre 2011 a 2016, para o município de Sinop-MT.

Na Figura 6 observa-se os valores médios mensais da radiação global medida e estimada pelos modelos dos diferentes agrupamentos de dados, em conjunto com os comportamentos mensais dos indicativos estatísticos. Nesse caso, destaca-se que estimativas de $\mathrm{H}$ utilizando coeficientes obtidos com diferentes agrupamentos não são significativamente diferentes. Durante o período seco (maio a setembro), os valores de $n / N$ tendem a ser elevados (próximos de 1,0), aumentado a dispersão de dados, e consequentemente, piorando os ajustes baseados em $\mathrm{R}^{2}$. Nesse período, o agrupamento anual, permitiu melhores ajustes quando comparados com os agrupamentos sazonais (estações do ano ) e mensais, confirmando a influência dos valores elevados de $\mathrm{n} / \mathrm{N}$ em meses com grandes percentuais de dias com céu aberto (SOUZA et al. 2016a).

As médias mensais de $\mathrm{H}$ medida variaram de 12,89 a $17,77 \mathrm{MJ} \mathrm{m}^{-2} \mathrm{dia}^{-1}$ (março e agosto), com médias anuais de $15,71 \mathrm{MJ} \mathrm{m}^{-2}$ dia $^{-1}$, enquanto que, a média anual de $\mathrm{H}$ estimada foi de $16,46 \pm 0,1 \mathrm{MJ} \mathrm{m}^{-2} \mathrm{dia}^{-1}$. Esses valores estão abaixo das estimativas obtidas por Souza et al. (2017) com modelos simplificados baseados na amplitude térmica diária $\left(21,03 \mathrm{MJ} \mathrm{m^{-2 }} \mathrm{dia}^{-1}\right)$, indicando o emprego de $\mathrm{n} / \mathrm{N}$ permite melhores estimativas de $\mathrm{H}$ na região de Sinop-MT. Independentemente do agrupamento de dados, foram obtidas supertimativas de $\mathrm{H}$ com base em $n / N$, com exceção de fevereiro (mês mais chuvoso da região (SOUZA et al., 2013). 

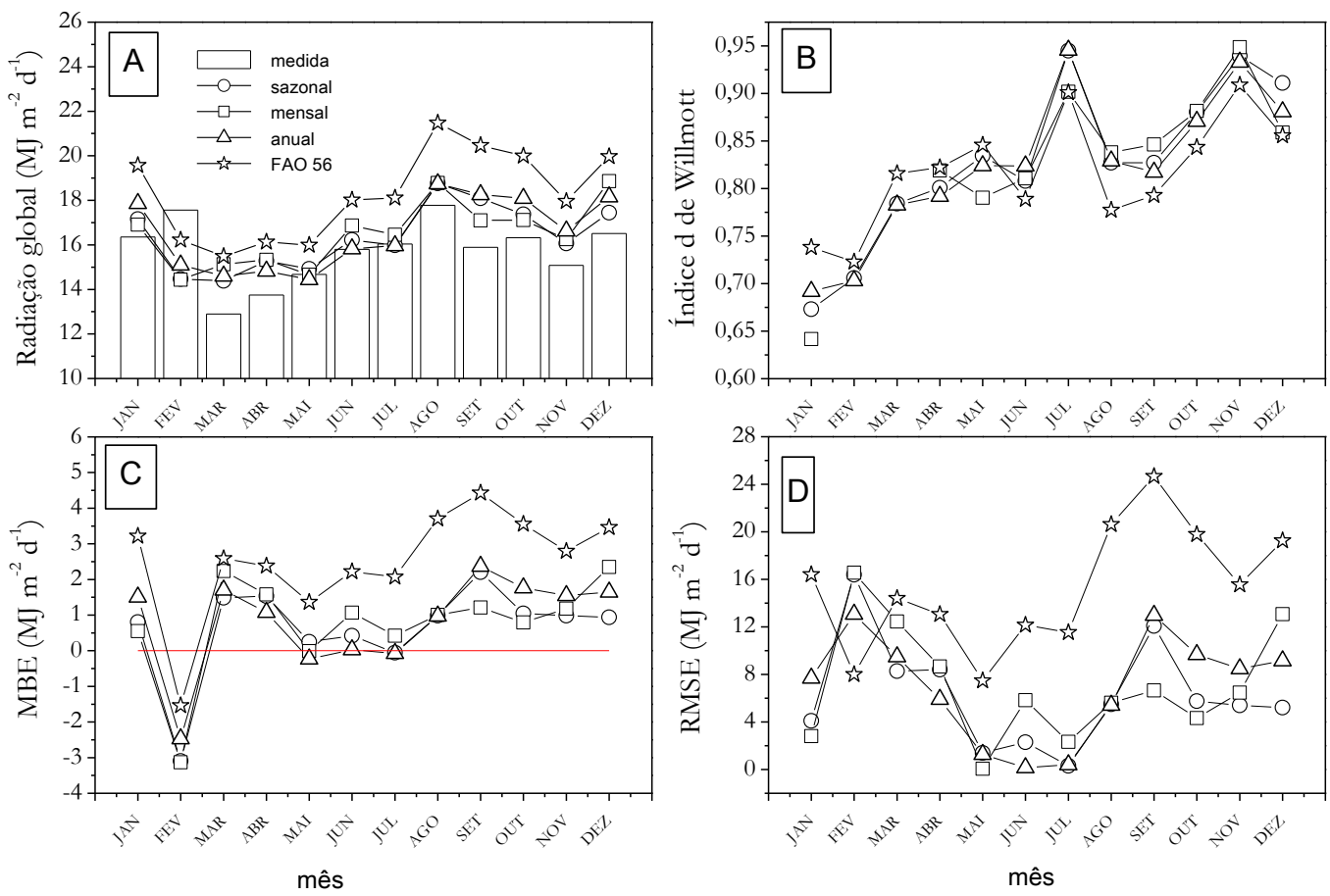

Figura 6 - Indicativos estatísticos médios mensais da radiação global $(\mathrm{H})$ medida e estimada por coeficientes de Angström-Prescott, em Sinop-MT.

Na Figura 7 observa-se os valores médios mensais da evapotranspiração de referência (ETo) estimadas com H medida e estimada com coeficientes "a" e " $b$ " calibrados para diferentes agrupamentos de dados. As médias mensais da evapotranspiração de referência (ETo) estimada por meio da radiação medida, variaram de 2,50 a 3,46 $\mathrm{mm} \mathrm{dia}^{-1}$ (junho e outubro) com média anual de 2,90 $\mathrm{mm}$ dia-1. Por outro lado, a ETo média obtida através da radiação global estimada nos diferentes agrupamentos foi de 3,05 $\pm 0,02 \mathrm{~mm} \mathrm{dia}^{-1}$, corroborando com Tanaka et al. (2016), que encontraram superestimativas dos valores de ETo por modelos simplificados para o estado de Mato Grosso.

Souza et al. (2016a) que encontraram para a região de Sinop, ETo média anual de $3,57 \mathrm{~mm} \mathrm{dia}^{-1}$ estimadas por Penman-Monteith quando a radiação global foi obtida por modelos simplificados de amplitude térmica. Nesse caso, nota-se novamente na ETo, que no período seco (maio a setembro), o uso de coeficientes obtidos com o agrupamento anual, permitiu melhores desempenhos estatísticos, permitindo recomendar a sua aplicação para projetos hidroagrícolas voltados para suplementação hídrica. A utilização de apenas uma equação simplificada ao longo do ano, também permite maior aplicabilidade por parte de diferentes usuários, visto que não demanda alterações periódicas dos coeficientes em suas estimativas. 

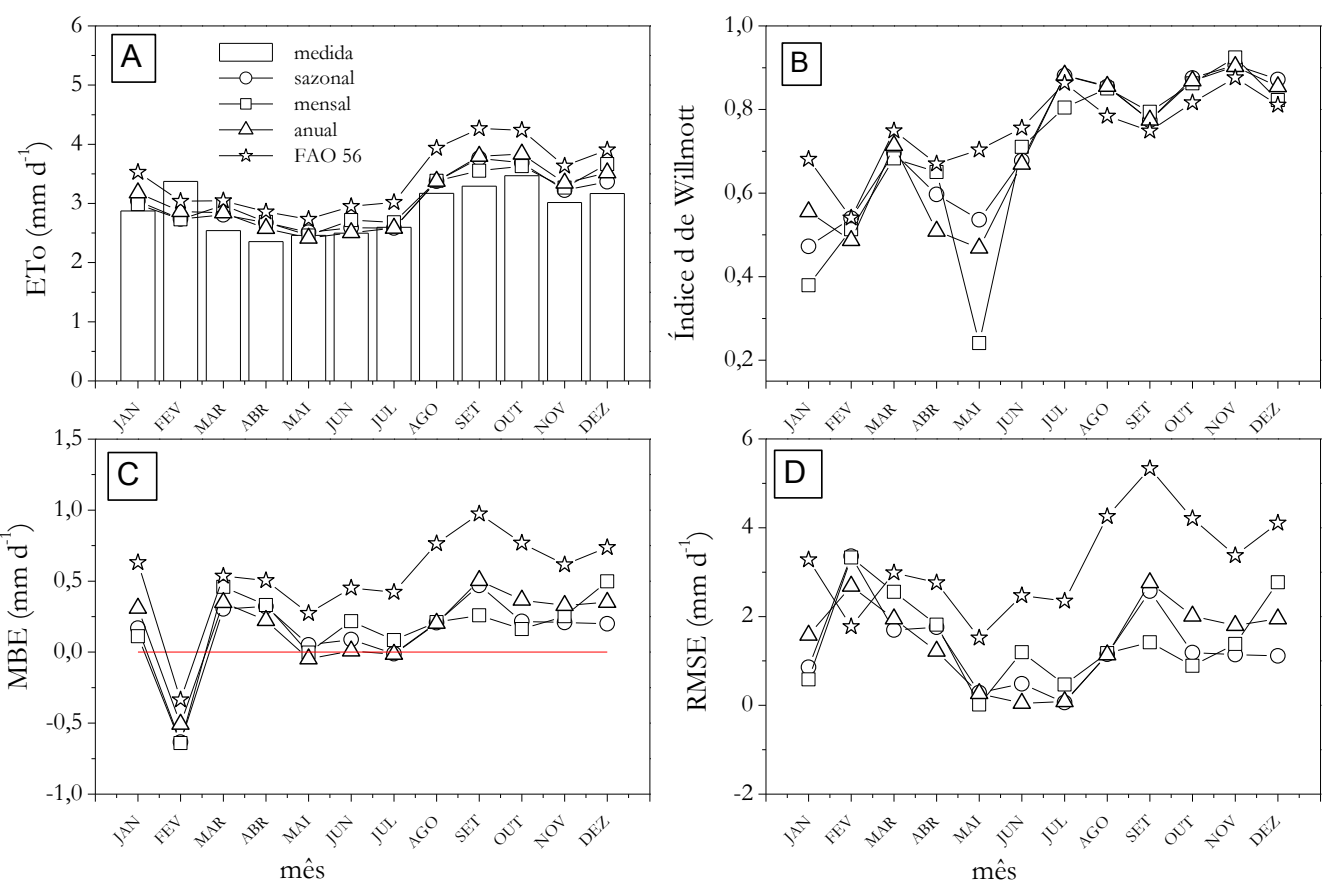

Figura 7 - Indicativos estatísticos médios mensais da evapotranspiração de referência (ETo) obtida por Penman-Monteith com a radiação global medida e estimada por coeficientes de Angström-Prescott, em Sinop-MT.

\section{CONCLUSÕES}

O uso de diferentes agrupamentos de dados (mensal, sazonal e anual) para os coeficientes da equação de Angström-Prescott na região de Sinop-MT, não geram diferenças nas estimativas da radiação global $(\mathrm{H})$ e da evapotranspiração de referência (ETo). Portanto, recomenda-se a utilização dos coeficientes linear e angular iguais a 0,2441 e 0,3774, respectivamente, e suas aplicações na estimativa da ETo pelo método de Penman-Monteith FAO 56.

\section{AGRADECIMENTOS}

O presente trabalho foi realizado com apoio da Coordenação de Aperfeiçoamento de Pessoal de Nível Superior - Brasil (CAPES) - Código de Financiamento 001; e da Fundação de Amparo à Pesquisa do Estado de Mato Grosso (Fapemat).

\section{REFERÊNCIAS BIBLIOGRÁFICAS}

ALLEN, R.G.; PEREIRA, L.S.; RAES, D.; SMITH, M. Crop evapotranspiration guidelines for computing crop water requirements. Rome: FAO, 1998. 297p. (Irrigation and drainage paper, 56).

ANDRADE, R.C.; TIBA, C. Extreme global solar irradiance due to cloud enhancement in northeastern Brazil. Renewable Energy, v. 86, p.1433-1441, 2016. 
ANGSTRÖM, A. Solar and terrestrial radiation. Quarterly Journal of the Royal Meteorological Society, v.50, p.121-126, 1924.

BADESCU, V. Assessing the performance of solar radiation computing models and model selection procedures. Journal of Atmospheric and Solar-Terrestrial Physics, v.105-106, p.119-134, 2013.

BAYRAKÇL, H.C.; DERNIRCAN, C.; KEÇEBAS, A.; The development of empirical models for estimating global solar radiation on horizontal surfasse: A case study. Renewable and Sustainable Energy Reviews, v.81, p.2771-2782, 2017.

BIUDES, M.S.; VOURLITIS, G.L.; MACHADO, N.G.; ARRUDA, P.H.Z.; NEVES, G.A.R.; LOBO, F.A.; NEALE, C.M.U.; NOGUEIRA, J.S. Patterns of energy exchange for tropical ecosystems across a climate gradiente in Mato Grosso, Brazil. Agricultural and Forest Meteorology, v.202, p.112-124, 2015.

BLANCO, F.F.; SENTELHAS, P.C. Coeficientes da equação de Angströn-Prescott para estimativa da insolação para Piracicaba, SP. Revista Brasileira de Agrometeorologia, v.10, n.2, p.295-300, 2002.

CARVALHO, D.F.; SILVA, D.G.; SOUZA, A.P.; GOMES, D.P.; ROCHA, H.S. Coeficientes da equação de Angström-Prescott e sua influência na evapotranspiração de referência em Seropédica, RJ. Revista Brasileira de Engenharia Agrícola e Ambiental, v.15, n.8, p.838-844, 2011.

CHAÂBANE, M.; MASMOUDI, M.; MEDHIOUB, K. Determination of Linke turbidity fator from solar radiation measurement in northern Tunisia. Renewable Energy, v.29, p.2065-2076, 2004.

DALLACORT, R.; FRETITAS, P. S. L.; GONÇALVES, A. C. A.; REZENDE, R.; BERTOLONHA, A.; SILVA, F. F.; TRINTINALHA, M. Determinação dos coeficientes da equação de Angstron para a região de Pelotina, estado do Paraná. Acta Scientiarum Agronomy, v.26, n.3, p.329-336, 2004.

DORNELAS, K.D.S.; SILVA, C.L.; OLIVEIRA, C.A.S. Coeficientes médios da equação de Angström-Prescott, radiação solar e evapotranspiração de referência em Brasília. Pesquisa Agropecuária Brasileira, v.41, n.8, p.1213-1219, 2006.

INMET. Instituto Nacional de Meteorologia. Disponível em: < http://www.inmet.gov.br/portal/index.php?r=home2/page\&page=estacoesDoAn o > . Acesso em:19 de março de 2018.

ISES. International Solar Energy Society. Units and symbols in solar energy. Solar Energy, v.61, n.1, p.3-4, 1997.

CAMPELO JÚNIOR, J.H. Relação sazonal entre radiação solar global e insolação no Sudeste da amazônia. Revista Brasileira de Agrometeorologia, v.6, n. , p.193-199, 1998.

LIU, X.; LI, Y.; ZHONG, X.; ZHAO, C.; JENSEN, J.R.; ZHAO, Y. Towards increasing availability of the Angström-Prescott radiation parameters across China: Spatial trend and modeling. Energy Conversion and Management, n.87, p.975-989, 2014.

LIU, X.; MEI, X.; LI, Y.; WANG, Q.; ZHANG, Y.; PORTER, J.R. Variation in reference crop evapotranspiration caused by the Angstrom-Prescott coefficient: Locally calibrated versus the FAO recommended. Agricultural Water Management, v.96, p.1137-1145, 2009. 
ÖZTOPAL, A.; SEN, Z. Terrestrial irradiation-sunshine duration clustering and prediction. Energy Conversion and Management, v.44, p.2159-2174, 2003.

PALÁCIOS, R.S.; SALLO, F.S.; MARQUES, J.B.; SANTOS, A.C.A.; MENEZES, J.A.; BIUDES, M.S.; NOGUEIRA, J.S. Variabilidade espaço-temporal da profundidade ótica de aerossóis nos biomas cerrado e pantanal da região central do Brasil. Nativa, v.6, n.1, p.56-65, 2018.

PAULESCU, M.; STEFU, N.; CALINOIU, D.; PAULESCU, E.; POP, N.; BOATA, R.; MARES, O. Angström-Prescott equation: physical basis, empirical models and sensitiviy analysis. Renewable and Sutainable Energy Reviews, v.62, p.495-506, 2016.

PEREIRA, A.R.; SEDIYAMA, G.C.; NOVA, N.A.V. Evapotranspiração. Fundag (Fundação de Apoio à Pesquisa Agrícola). Campinas-SP, 2013. 323p.

PRESCOTT, J.A. Evaporation from a water surface in relation to solar radiation. Transactions of the royal society of south Australia, v.64, p.114-118, 1940.

ROSSI, T.J.; ESCOBEDO, J.F.; SANTOS, C.M.; ROSSI, L.R.; SILVA, M.B.P.; DAL PAI, E. Global, diffuse and direct solar radiation of the infrared spectrum in Botucatu/SP/Brazil. Renewable and Sustainable Energy Reviews, n.82, p.448459, 2018.

SILVA, M.B.P.; ESCOBEDO, J.F.; ROSSI, T.J.; SANTOS, C.M.; SILVA, S.H.M.G. Performance of the Angstrom-Prescott Model (A-P) and SVW and ANN Techniques to estimate the daily global solar irradiation in Botucatu/SP/Brazil. Journal of Atmospheric and Solar-Terrestrial Physics, v.160, p.11-23, 2017.

SOUZA, A.P.; SILVA, A.C.; TANAKA, A.A.; ULIANA, E.M.; ALMEIDA, F.T.; KLAR, A.E.; GOMES, A.W.A. Global radiation by simplified models for the state of Mato Grosso, Brazil. Pesquisa Agropecuária Brasileira, v.52, n.4, p.215-227, 2017.

SOUZA, A.P.; TANAKA, A.A.; SILVA, A.C.; ULIANA, E.M.; ALMEIDA, F.T.; GOMES, A.W.A.; KLAR, A.E. Reference evapotranspiration by Penman-Monteith Fao 56 with missing data of global radiation. Brazilian Journal of Biosystems Engineering, v.10, n.2, p.217-233, 2016a.

SOUZA, A.P.; ZAMADEI, T.; MONTEIRO, E.B.; CASAVECCHIA, B.H. Transmissividade Atmosférica da Radiação Global na Região Amazônica de Mato Grosso. Revista Brasileira de Meteorologia, v.31, n.4, p.639-648, 2016b.

SOUZA, J.L.; LYRA, G.B.; SANTOS, C.M.; FERREIRA JUNIOR, R.A.; TIBA, C.; LYRA, G.B.; LEMES, M.A.M. Empirical models of daily and monthly global solar irradiation using Sunshine duration for Alagoas State, Northeastern Brazil. Sustainable Energy Technologies and Assessments, v.14, p.35-45, 2016c.

SOUZA, A.P.; ESCOBEDO, J.F. Estimativas da radiação global incidente em superfícies inclinadas com base na razão de insolação. Revista Brasileira de Ciências Agrárias, v.8, n.3, p.483-491, 2013.

SOUZA, A.P.; MOTA, L.L.; ZAMADEI, T.; MARTIM, C.C.; ALMEIDA, F.T.; PAULINO, J. Classificação climática e balanço hídrico climatológico para o estado de Mato Grosso. Nativa, v.1, n.1, p.34-43, 2013.

SOUZA, A.P.; ESCOBEDO, J.F.; DAL PAI, A.; GOMES, E.N. Estimativas das componentes da radiação solar incidente em superfícies inclinadas baseadas na 
radiação global horizontal. Revista Brasileira de Engenharia Agrícola e Ambiental, v.15, n.3, p.277288, 2011.

TANAKA, A.A.; SOUZA, A.P.; KLAR, A.E.; SILVA, A.C.; GOMES, A.W.A. Evapotranspiração de referência estimada por modelos simplificados para 0 Estado do Mato Grosso. Pesquisa Agropecuária Brasileira, v.51, n.2, p.91-104, 2016.

WILLMOTT, C.J.; CKLESON, S.G.; DAVIS, R.E.; FEDDEMA, J.J.; KLINK, K.M.; LEGATES, D.R.; O'DONNELL, J.; ROWE, C.M. Statistics for the evaluation and comparison of models. Journal of Geophysical Research, v.90, p.8995-9005, 1985 